\title{
Existence solution of neutral fractional differential equation inclusions with fractional operator
}

\author{
M. H.M. Rashid and A. Al-Omari
}




\title{
EXISTENCE SOLUTION OF NEUTRAL FRACTIONAL DIFFERENTIAL INCLUSIONS WITH FRACTIONAL OPERATOR
}

\author{
M. H. M. RASHID AND A. AL-OMARI
}

Received 08 June, 2011

\begin{abstract}
This paper is mainly concerned with existence of mild solutions for a first order impulsive neutral fractional integrodifferential inclusions with nonlocal initial conditions in $\alpha$ norm. We assume that the linear part generates an analytic semigroup and transform it into an integral inclusion. By using a fixed point theorem for multi-valued maps due to Dhage, a main existence theorem is established. Finally, we present an example to illustrate this main theorem.
\end{abstract}

2010 Mathematics Subject Classification: 34A60; 34A37; 34G20

Keywords: analytic compact semigroup, $\alpha$-norm, mild solution, impulsive integrodifferential equation, non-local condition, fixed point

\section{INTRODUCTION}

In recent years, impulsive differential and partial differential equations have become more important in some mathematical models of real phenomena, especially in control, biological, and medical domains. In these models, the investigated simulating processes and phenomena usually have short-time perturbations during their evolution. The perturbations are performed discretely and their duration is negligible in comparison with the total duration of the processes. That is why the perturbations are considered to take place instantaneously in the form of impulses. The theory of impulsive differential equations has seen considerable development; see the monographs of Bainov and Simeonov [1], Lakshmikantham et al. [12], and Samoilenko and Perestyuk [17], where numerous properties of their solutions are studied and detailed bibliographies are given.

The starting point of this paper is the work in paper [9], where the authors have investigated the existence of solutions for the neutral partial differential equations in $\alpha$-norm

$$
\begin{gathered}
\frac{d}{d t}\left[u(t)-F\left(t, u\left(h_{1}(t)\right)\right)\right]=-A\left[u(t)-F\left(t, u\left(h_{1}(t)\right)\right)\right]+G\left(t, u\left(h_{2}(t)\right)\right), \quad t \in J \\
u(0)+g(u)=u_{0} .
\end{gathered}
$$


Furthermore, in [5], authors studied the following impulsive neutral partial differential equations in $\alpha$-norm

$$
\begin{gathered}
\frac{d}{d t}\left[u(t)-F\left(t, u\left(h_{1}(t)\right)\right)\right] \in A u(t)+\int_{0}^{t} K(t, s) G\left(s, u\left(h_{2}(s)\right)\right) d s, \\
t \in J \backslash\left\{t_{1}, t_{2}, \cdots, t_{m}\right\}, \quad \text { where } J=[0, a], \\
\left.\Delta u\right|_{t_{k}}=I_{k}\left(u\left(t_{k}^{-}\right)\right), \quad k=1,2, \cdots, m . \\
u(0)=g(u) \in X .
\end{gathered}
$$

Motivated by the above-mentioned works, in this paper we study the existence of mild solutions for the following system in a general Banach space $X$ with $\alpha$-norm:

$$
\begin{gathered}
{ }^{c} D_{a^{+}}^{q}\left[u(t)-F\left(t, u\left(h_{1}(t)\right)\right)\right] \in-A u(t)+\int_{0}^{t} K(t, s) G\left(s, u\left(h_{2}(s)\right)\right) d s, \\
t \in J \backslash\left\{t_{1}, t_{2}, \cdots, t_{m}\right\}, \quad \text { where } J=[0, a], 0<q \leq 1, \\
\left.\Delta u\right|_{t_{k}}=I_{k}\left(u\left(t_{k}^{-}\right)\right), \quad k=1,2, \cdots, m . \\
u(0)+g(u)=u_{0} \in X .
\end{gathered}
$$

Here $A$ is the infinitesimal generator of a compact, analytic semigroup

$\{T(t), t>0\}, G$ is a multi-valued map and $\left.\Delta u\right|_{t=t_{k}}=u\left(t_{k}^{+}\right)-u\left(t_{k}^{-}\right)$, where $u\left(t_{k}^{+}\right)$and $u\left(t_{k}^{-}\right)$represent the right and left limits of $u(t)$ at $t=t_{k}$. Let $K: D \rightarrow \mathbb{R}$, $D=\{(t, s) \in J \times J: 0 \leq s \leq t\}$ and $F, G, g, I_{k}(k=1,2, \cdots, m)$ and $h_{1}, h_{2}$ are given functions to be specified later.

The nonlocal Cauchy problem was considered by Byszewski [2] and the importance of nonlocal conditions in different fields has been discussed in $[2,7]$ and the references therein. For example, in [7] the author described the diffusion phenomenon of a small amount of gas in a transparent tube by using the formula

$$
g(u)=\sum_{j=0}^{p} c_{j} u\left(t_{j}\right),
$$

where $c_{j}, j=0,1, \cdots, p$ are given constants and $0<t_{0}<t_{1}<\cdots<t_{p}<a$. In this case the above equation allows the additional measurement at $t_{j}, j=0,1, \cdots, p$. In the past several years theorems about existence, uniqueness and stability of differential and functional differential abstract evolution Cauchy problem with nonlocal conditions have been studied by Byszewski and Lakshmikantham [4], by Byszewski [2,3], by Fu [10], by Fu and Ezzinbi [11] and recently, Cheng et al. [5] studied the existence results for impulsive neutral integrodifferential equations with nonlocal conditions. 


\section{PRELIMINARIES}

In this section, we introduce some definitions, notations and preliminary facts from multi-valued analysis which are used throughout this paper.

Let $(X,\|\|$.$) be a Banach space. \mathcal{C}(J, X)$ is the Banach space of continuous functions from $J$ to $X$ with the norm $\|u\|_{J}=\sup \{u(t): t \in J\} . B(X)$ denotes the Banach space of bounded linear operators from $X$ to $X$, with norm $\|N\|_{B(X)}=$ $\sup \{\|N(x)\|:\|x\|=1\}$. A measurable function $v: J \rightarrow X$ is Bochner integrable if and only if $\|v\|$ is Lebesgue integrable (for properties of the Bochner integral see Yosida [18]). $L^{1}(J, X)$ denotes the Banach space of Bochner integrable functions $v: J \rightarrow X$ with norm $\|v\|_{L^{1}}=\int_{0}^{a}\|v(t)\| d t$ for all $x \in L^{1}(J, X)$

We use the notations $\mathcal{P}(X)$ for the family of all subsets of $X$. Let $P_{b, c l}(X)$ and $P_{c p, c}(X)$ denote respectively the classes of all bounded-closed and compact-convex subsets of $X$. Similarly, $P_{b c c}(X)$ denotes the classes of all bounded, closed and convex subsets of $X$. A multi-valued map $G: X \rightarrow P(X)$ is convex (closed) for all $x \in X$. G is said to be bounded on bounded sets if $G(B)=\bigcup_{x \in B} G(x)$ is bounded in $X$ for all $B \in P_{b}(X)$ (i.e.,

$\left.\sup _{x \in B}\{\sup \{\|y\|: y \in G(x)\}\}<\infty\right)$. $G$ is called upper semicontinuous (u.s.c.) on $X$ if for each $x_{0} \in X$ the set $G\left(x_{0}\right)$ is a nonempty, closed subset of $X$, and if for each open subset $U$ of $X$ containing $G\left(x_{0}\right)$, there exists an open neighborhood $\Gamma$ of $x_{0}$ such that $G(\Gamma) \subset \mathcal{U}$.

$G$ is said to be completely continuous if $G(B)$ is relatively compact for every $B \in P_{b}(X)$. If the multi-valued map $G$ is completely continuous with nonempty compact values, then $G$ is u.s.c. if and only if $G$ has a closed graph, i.e., $x_{n} \rightarrow$ $x_{*}, y_{n} \rightarrow y_{*}, y_{n} \in G\left(x_{n}\right)$ imply $y_{*} \in G\left(x_{*}\right)$. We say that $G$ has a fixed point if there is $x \in X$ such that $x \in G(x)$.

A multi-valued map $G: J \rightarrow P_{c l}(X)$ is said to be measurable if for each $x \in X$ the function $Y: J \rightarrow \mathbb{R}$ defined by $Y(t)=d(x, G(t))=\inf \{\|x-z\|: z \in G(t)\}$ is measurable. For more details on multi-valued maps we refer to the book by Deimling [6].

Let $-A$ be the infinitesimal generator of an analytic semigroup $\{T(t), t \geq 0\}$ in $X$. We note that if $-A$ is the infinitesimal generator of an analytic semigroup then $-(A+\alpha I)$ is invertible and generates a bounded analytic semigroup for $\alpha>0$ large enough. This allows us to reduce the general case in which $-A$ is the infinitesimal generator of an analytic semigroup to the case in which the semigroup is bounded and the generator is invertible. Hence for convenience, we suppose that $\|T(t)\| \leq M$ for $t \geq 0$.

We assume without loss of generality that $0 \in \rho(-A)$ where $\rho(-A)$ is the resolvent set of $-A$. This allows us to define the fractional power $A^{\alpha}$ for $0<\alpha<1$, as a closed linear operator on its domain $D\left(A^{\alpha}\right)$ with inverse $A^{-\alpha}$. We have the following basic properties $A^{\alpha}$. 
Theorem 1 ([16]).

(1) $X_{\alpha}=D\left(A^{\alpha}\right)$ is a Banach space with norm $\|x\|_{\alpha}=\left\|A^{\alpha} x\right\|$ for $x \in D\left(A^{\alpha}\right)$.

(2) $T(t): X \rightarrow X_{\alpha}$ for each $x>0$.

(3) $A^{\alpha} T(t) x=T(t) A^{\alpha} X$ for each $x \in D\left(A^{\alpha}\right)$ and $t>0$.

(4) For every $t>0, A^{\alpha} T(t)$ is bounded on $X$ and there exist $C_{\alpha}>0$ and $\delta>0$ such that

$$
\left\|A^{\alpha} T(t)\right\| \leq C_{\alpha} t^{-\alpha} e^{-\delta t} \leq C_{\alpha} t^{-\alpha} .
$$

(5) $A^{-\alpha}$ is a bounded linear operator in $X$ with $D\left(A^{\alpha}\right)=i m\left(A^{-\alpha}\right)$.

(6) If $0<\alpha \leq \beta$, then $D\left(A^{\beta}\right) \hookrightarrow D\left(A^{\alpha}\right)$.

Remark 1. Observe as in [14] that by Theorem 1 (2) and (3), the restriction $T_{\alpha}(t)$ of $T(t)$ to $X_{\alpha}$ is exactly the part of $T(t)$ in $X_{\alpha}$. Let $x \in X_{\alpha}$. Since

$$
\|T(t) x\|_{\alpha}=\left\|A^{\alpha} T(t) x\right\|=\left\|T(t) A^{\alpha} x\right\| \leq\|T(t)\|\left\|A^{\alpha} x\right\|=\|T(t)\|\|x\|_{\alpha}
$$

and as $t$ decreases to 0

$$
\|T(t) x-x\|_{\alpha}=\left\|A^{\alpha} T(t) x-A^{\alpha} x\right\|=\left\|T(t) A^{\alpha} x-A^{\alpha} x\right\| \rightarrow 0
$$

for all $x \in X_{\alpha}$ it follows that $\left(T(t)_{t \geq 0}\right)$ is a family of strongly continuous semigroup on $X_{\alpha}$ and $\left\|T_{\alpha}(t)\right\| \leq\|T(t)\|$ for all $t \geq 0$.

To begin with the analysis we need some basic definitions and properties from the fractional calculus theory.

Definition 1. A real function $f(x), x \geq 0$ is said to be space $C_{\mu}, \mu \in \mathbb{R}$ if there exist a real number $p(>\mu)$, such that $f(x)=x^{p} f_{1}(x)$, where $f_{1} \in C[0, \infty)$ and it is said to be in the space $C_{\mu}^{m}$ if and only if $f^{(m)} \in C_{\mu}, m \in \mathbb{N}$.

Definition 2. The Riemann-Liouville fractional integral operator of order $0 \leq \alpha$, of a function $f \in C_{\mu}, \mu \geq-1$ is defined as

$$
\begin{aligned}
I^{\alpha} f(x) & =\frac{1}{\Gamma(\alpha)} \int_{0}^{x}(x-t)^{\alpha-1} f(t) d t, \quad \alpha>0, x>0, \\
I^{0} f(x) & =f(x) .
\end{aligned}
$$

While modeling some real world phenomena with fractional differential equation the Riemann-Liouville derivative has certain disadvantage. Therefore, we shell introduce a modified fractional differential operator $D_{*}^{\alpha}$ proposed by M. Caputo.

Definition 3. The fractional derivative of $f(x)$ in the Caputo sense is defined as

$$
\begin{aligned}
D I_{x}^{\alpha} f(x)= & I^{m-\alpha} D^{m} f(x) \\
= & \frac{1}{\Gamma(m-\alpha)} \int_{0}^{x}(x-t)^{m-\alpha-1} D^{m} f(t) d t, \\
& \quad \text { for } \quad m-1 \leq \alpha<m, m \in \mathbb{N}, x>0, f \in C_{-1}^{m} .
\end{aligned}
$$


In order to define the solution of system (1.1) we shall consider the space $\Omega=\left\{u:[0, a] \rightarrow X_{\alpha}: u \in \mathcal{C}\left(\left(t_{k-1}, t_{k}\right], X\right), k=1,2, \cdots, m\right.$ and there exist

$u\left(t_{k}^{-}\right)$and $u\left(t_{k}^{+}\right), k=1,2, \cdots, m$

with $\left.u\left(t_{k}^{-}\right)=u\left(t_{k}\right), u(0)+g(u)=u_{0}\right\}$.

which is a Banach space with the norm

$$
\|u\|_{\Omega}=\sup _{t \in J}\|u(t)\|_{\alpha} .
$$

Now we define the mild solution for the system (1.1).

Definition 4. A continuous function $u$, such that

(i) $u(0)+g(u)=u_{0}$ for each $0 \leq t \leq a$;

(ii) $\left.\Delta u\right|_{t=t_{k}}=I_{k}\left(u\left(t_{k}^{-}\right)\right), k=1,2, \cdots, m$, the restriction of $u($.$) to the interval$ $[0, a) \backslash\left\{t_{1}, t_{2}, \cdots, t_{k}\right\}$ is continuous.

satisfying the integro-differential equation

$$
\begin{aligned}
u(t) & =T(t)\left[u_{0}-g(u)-F\left(0, u\left(h_{1}(0)\right)\right)\right]+F\left(t, u\left(h_{1}(t)\right)\right) \\
& +\frac{1}{\Gamma(q)} \int_{0}^{t}(t-s)^{q-1} T(t-s) F\left(s, u\left(h_{1}(s)\right)\right) d s \\
& +\frac{1}{\Gamma(q)} \int_{0}^{t}(t-s)^{q-1} T(t-s) \int_{0}^{s} K(s, w) v(w) d w d s \\
& +\sum_{k=1}^{m} T\left(t-t_{k}\right) I_{k}\left(u\left(t_{k}^{-}\right)\right), \quad t \in J, v(t) \in G\left(t, u\left(h_{2}(t)\right)\right)
\end{aligned}
$$

is called a mild solution of problem (1.1).

Our main results are based on the following lemmas.

Lemma 1 ([13]). Let $X$ be a Banach space. Let $G: J \times X \rightarrow P_{b, c l, c}(X)$ satisfied that

(i) For each $u \in X,(t, u) \rightarrow G(t, u)$ is measurable with respect to $t$;

(ii) For each $t \in J,(t, u) \rightarrow G(t, u)$ is u.s.c. with respect to $u$;

(iii) For each fixed $u \in \mathcal{C}(J, X)$, the set

$$
S_{G, u}=\left\{v \in L^{1}(J, X): v(t) \in G\left(t, u\left(h_{2}(t)\right)\right) \text { for a.e } t \in J\right\}
$$

is nonempty. Let $\Gamma$ be a linear continuous mapping from $L^{1}(J, X)$ to $C(J, X)$. Then the operator

$$
\Gamma \circ S_{G}: \mathcal{C}(J, X) \rightarrow P_{c p, c}(\mathcal{C}(J, X)), \quad u \rightarrow\left(\Gamma \circ S_{G}\right)(u):=\Gamma\left(S_{G, u}\right)
$$

is a closed graph operator in $\mathcal{C}(J, X) \times \mathcal{C}(J, X)$. 
Lemma 2 ([8]). Let $B(0, r)$ and $B[0, r]$ denote respectively the open and closed balls in a Banach space $X$ centered at the origin and of radius $r$ and let $\mathcal{A}: X \rightarrow$ $P_{b c c}(X)$ and $B: B[0, r] \rightarrow P_{c p, c}(X)$ be two multi-valued operators satisfying:

(a) A is a multi-valued contraction, and

(b) $\mathcal{B}$ is upper semicontinuous and completely continuous. Then either

(i) the operator inclusion $u \in \mathcal{A} u+\mathscr{B} u$ has a solution in $B[0, r]$, or

(ii) there exists a $w \in X$ with $\|w\|=r$ such that $\delta w \in \mathcal{A} w+\mathscr{B} w$ for some $\delta>1$.

We have the following result from [14].

Lemma 3. $T(t)_{t \geq 0}$ is an immediately compact semigroup in $X_{\alpha}$, and hence it is immediately norm-continuous.

\section{MAIN RESULT}

In this section, we state and prove the existence theorem for the system (1.1). Let us list the following hypothesis: for some $\alpha \in(0,1)$,

(H1) There exists a constant $\beta \in(0,1)$ with $\alpha \leq \beta \leq 1$ such that $F:[0, a] \times$ $X_{\alpha} \rightarrow X_{\beta}$ is a continuous function, and $A^{\bar{\beta}} F:[0, a] \times X_{\alpha} \rightarrow X_{\alpha}$ satisfies the Lipschitz condition, that is, there exists a constant $L>0$ such that

$$
\left\|A^{\beta} F\left(t_{1}, u_{1}\right)-A^{\beta} F\left(t_{2}, u_{2}\right)\right\| \leq L\left(\left|t_{1}-t_{2}\right|+\left\|u_{1}-u_{2}\right\|_{\alpha}\right),
$$

for any $\left(t_{1}, u_{1}\right),\left(t_{2}, u_{2}\right) \in[0, a] \times X_{\alpha}$. Moreover, there exists a constant $L_{1}>$ 0 such that the inequality

$$
\left\|A^{\beta} F(t, u)\right\|_{\alpha} \leq L_{1}\left(\|u\|_{\Omega}+1\right)
$$

holds for any $u \in X_{\alpha}$ with

$$
L_{0}=\left[(M+1) L+\frac{L M_{\alpha-\beta} a^{q+\alpha-\beta}}{\Gamma(q)(q+\alpha-\beta)}\right]<1 .
$$

(H2) The multi-valued map $G: J \times X_{\alpha} \rightarrow P_{c, c p}(X)$ satisfies the following conditions:

(i) For each $t \in J$, the function $G(t,):. X_{\alpha} \rightarrow P_{c, c p}(X)$ is u.s.c.; and for each $x \in X_{\alpha}$, the function $G(., u)$ is measurable. And for each fixed $u \in \Omega$ the set

$$
S_{G, u}=\left\{v \in L^{1}(J, X): v(t) \in G(t, u) \quad \text { for a.e } t \in J\right\}
$$

is nonempty.

(ii) For each positive number $l>0$, there exists a positive function $w(l)$ dependent on $l$ such that

$$
\sup _{\|u\|_{\alpha} \leq l}\|G(t, u)\| \leq w(l),
$$


where

$$
\|G(t, u)\|=\sup \{\|v\|: v \in G(t, u)\}, \quad\|u\|_{\alpha}=\sup _{0 \leq s \leq a}\|u(s)\|_{\alpha} .
$$

(H3) $h_{i} \in \mathscr{C}(J, J), i=1,2 . g: \Omega \rightarrow X_{\alpha}$ satisfies that

(i) There exist positive constants $L_{2}$ and $L_{3}$ such that

$$
\|g(u)\|_{\alpha}<L_{2}\|u\|_{\Omega}+L_{3} \quad \text { for all } u \in \Omega .
$$

(ii) $g$ is a completely continuous map.

(H4) For each $t \in J, K(t, s)$ is measurable on $J$ and

$$
K(t)=e s s \sup \{|K(t, s)|, 0 \leq s \leq t\}
$$

is bounded on $J$. The map $t \rightarrow K_{t}$ is continuous from $J$ to $L^{\infty}(J, \mathbb{R})$, here $K_{t}(s)=K(t, s)$ and $K=\sup _{t \in J} K(t)$.

(H5) $I_{k} \in \mathscr{C}\left(X_{\alpha}, X_{\alpha}\right), k=1,2, \cdots, m$, and there exist nondecreasing functions $\psi_{k} \in \mathcal{C}\left(J, \mathbb{R}_{+}\right), k=1,2, \cdots, m$ such that

$$
\left\|I_{k}(u)\right\|_{\alpha} \leq \psi_{k}\left(\|u\|_{\alpha}\right) \leq N\|u\|_{\Omega}, \quad \text { for } u \in X_{\alpha}, N \in \mathbb{R}_{+}
$$

(H6) There exists a real number $r>0$ such that

$$
r \leq \frac{2+M\left(\left\|u_{0}\right\|_{\alpha}+L_{3}\right)+\frac{M_{\alpha} a^{q-\alpha}(1+a w(r) K)}{(q-\alpha) \Gamma(q)}}{1-\left[M\left(L_{2}+m N\right)+2 L_{1}+\frac{M_{\alpha} L_{1} a^{q-\alpha}}{(q-\alpha) \Gamma(q)}\right]},
$$

where

$$
\left[M\left(L_{2}+m N\right)+2 L_{1}+\frac{M_{\alpha} L_{1} a^{q-\alpha}}{(q-\alpha) \Gamma(q)}\right]<1 .
$$

Theorem 2. Let $u_{0} \in X_{\alpha}$. If the hypotheses $(H 1)-(H 6)$ are satisfied, then the system (1.1) admits at least one mild solution on $J$.

Proof. Consider the operator $N: \Omega \rightarrow \mathcal{P}(\Omega)$ defined by

$$
\begin{aligned}
N(u) & =\left\{w \in \Omega: w(t)=T(t)\left[u_{0}-g(u)-F\left(0, u\left(h_{1}(0)\right)\right)\right]+F\left(t, u\left(h_{1}(t)\right)\right)\right. \\
& +\frac{1}{\Gamma(q)} \int_{0}^{t}(t-s)^{q-1} T(t-s) F\left(s, u\left(h_{1}(s)\right)\right) d s \\
& +\frac{1}{\Gamma(q)} \int_{0}^{t}(t-s)^{q-1} T(t-s) \int_{0}^{s} K(s, w(s) v(w) d w d s \\
& \left.+\sum_{k=1}^{m} T\left(t-t_{k}\right) I_{k}\left(u\left(t_{k}^{-}\right)\right), \quad t \in J, v(t) \in G\left(t, u\left(h_{2}(t)\right)\right)\right\} .
\end{aligned}
$$

Clearly the fixed point of $N$ are solutions of the system (1.1). Let

$$
\mathcal{A}(u)=F\left(t, u\left(h_{1}(t)\right)\right)-T(t) F\left(0, u\left(h_{1}(0)\right)\right)
$$




$$
+\frac{1}{\Gamma(q)} \int_{0}^{t}(t-s)^{q-1} T(t-s) F\left(s, u\left(h_{1}(s)\right)\right) d s
$$

and

$$
\begin{aligned}
\mathscr{B}(u) & =\left\{w \in \Omega: w(t)=T(t)\left[u_{0}-g(u)\right]\right. \\
& +\frac{1}{\Gamma(q)} \int_{0}^{t}(t-s)^{q-1} T(t-s) \int_{0}^{s} K(s, w(s) v(w) d w d s \\
& \left.+\sum_{k=1}^{m} T\left(t-t_{k}\right) I_{k}\left(u\left(t_{k}^{-}\right)\right), v \in S_{G, u}\right\} .
\end{aligned}
$$

In order to apply Lemma 2, we give the proof in several steps.

Step 1. A is a contraction.

Take $x, y \in B_{l}$. Then for each $t \in J$ and by condition (H1), we have that

$$
\begin{aligned}
& \|\mathcal{A}(x)(t)-\mathcal{A}(y)(t)\|_{\alpha} \\
& \leq\left\|F\left(t, x\left(h_{1}(t)\right)\right)-F\left(t, y\left(h_{1}(t)\right)\right)\right\|_{\alpha}+\left\|T(t)\left[F\left(0, x\left(h_{1}(0)\right)\right)-F\left(0, y\left(h_{1}(0)\right)\right)\right]\right\|_{\alpha} \\
& +\left\|\frac{1}{\Gamma(q)} \int_{0}^{t}(t-s)^{q-1} T(t-s) F\left(t, x\left(h_{1}(t)\right)\right)-F\left(t, y\left(h_{1}(t)\right)\right)\right\|_{\alpha} \\
& =\left\|A^{\alpha}\left[F\left(t, x\left(h_{1}(t)\right)\right)-F\left(t, y\left(h_{1}(t)\right)\right)\right]\right\| \\
& +\left\|A^{\alpha} T(t)\left[F\left(0, x\left(h_{1}(0)\right)\right)-F\left(0, y\left(h_{1}(0)\right)\right)\right]\right\| \\
& +\left\|\frac{1}{\Gamma(q)} \int_{0}^{t}(t-s)^{q-1} A^{\alpha-\beta} T(t-s) A^{\beta}\left[F\left(t, x\left(h_{1}(t)\right)\right)-F\left(t, y\left(h_{1}(t)\right)\right)\right] d s\right\| \\
& \leq\left[(M+1) L+\frac{L M_{\alpha-\beta} a^{q+\alpha-\beta}}{\Gamma(q)(q+\alpha-\beta)}\right] \sup _{s \in J}\|x(s)-y(s)\|_{\alpha} \\
& =\left[\begin{array}{l}
L_{0} \sup _{s \in J}\|x(s)-y(s)\|_{\alpha} .
\end{array}\right.
\end{aligned}
$$

Thus,

$$
\|\mathcal{A}(x)-\mathcal{A}(y)\| \leq L_{0}\|x-y\|_{\alpha} .
$$

Therefore, by assumption $0<L_{0}<1$, we can see that $\mathcal{A}$ is a contraction.

Step 2. $\mathscr{B}(u)$ is convex for each $u \in \Omega$.

Indeed, if $w_{1}, w_{2} \in \mathcal{B}(x)$, then there exist $v_{1}, v_{2} \in S_{G, x}$, such that for each $t \in J$ we have

$$
\begin{aligned}
w_{i} & =T(t)\left[u_{0}-g(u)\right]+\sum_{k=1}^{m} T\left(t-t_{k}\right) I_{k}\left(u\left(t_{k}^{-}\right)\right) \\
& +\frac{1}{\Gamma(q)} \int_{0}^{t}(t-s)^{q-1} T(t-s) \int_{0}^{s} K(s, w) v_{i}(w) d w d s, \quad i=1,2 .
\end{aligned}
$$


Let $0 \leq \lambda \leq 1$. Then for each $t \in J$ we have

$$
\begin{gathered}
\lambda w_{1}+(1-\lambda) w_{2}=T(t)\left[u_{0}-g(u)\right]+\sum_{k=1}^{m} T\left(t-t_{k}\right) I_{k}\left(u\left(t_{k}^{-}\right)\right) \\
+\frac{1}{\Gamma(q)} \int_{0}^{t}(t-s)^{q-1} T(t-s) \int_{0}^{s} K(s, w)\left[\lambda v_{1}(w)+(1-\lambda) v_{2}(w)\right] d w d s .
\end{gathered}
$$

Since $S_{G, u}$ is convex (because $G$ has convex values), then

$\lambda w_{1}+(1-\lambda) w_{2} \in N(u)$.

Step 3. $B$ is bounded on bounded sets of $\Omega$.

Let $B_{l}=\left\{u \in \Omega:\|u\|_{\alpha} \leq l\right\}$ be a bounded set in $\Omega$. Now for each $u \in$ $B_{l}, x \in \mathscr{B}(u)$, there exists a function $v \in S_{G, u}$ such that for each $t \in J$. We have

$$
\begin{aligned}
\|x(t)\|_{\alpha} & \leq\left\|T(t)\left(x_{0}-g(x)\right)\right\|_{\alpha} \\
& +\left\|\frac{1}{\Gamma(q)} \int_{0}^{t}(t-s)^{q-1} T(t-s) \int_{0}^{s} K(s, x) v(x) d w d s\right\|_{\alpha} \\
& +\sum_{k=1}^{m}\left\|T\left(t-t_{k}\right) I_{k}\left(x\left(t_{k}^{-}\right)\right)\right\|_{\alpha} \\
& \leq\|T(t)\|\left\|\left(x_{0}-g(x)\right)\right\|_{\alpha}+ \\
& \left\|\frac{1}{\Gamma(q)} \int_{0}^{t}(t-s)^{q-1} A^{\alpha} T(t-s) \int_{0}^{s} K(s, x) v(x) d w d s\right\| \\
& +\sum_{k=1}^{m}\left\|A^{\alpha} T\left(t-t_{k}\right) I_{k}\left(x\left(t_{k}^{-}\right)\right)\right\| \\
& \leq M\left(L_{2}\|x\|_{\Omega}+L_{3}+\left\|x_{0}\right\|_{\alpha}\right) \\
& +\frac{a w(l) \sup _{t \in J} K(t)}{\Gamma(q)} \int_{0}^{t} \frac{M_{\alpha}(t-s)^{q-1}}{(t-s)^{\alpha}} d s+M \sum_{k=1}^{m} \psi_{k}\left(\|x\|_{\alpha}\right) \\
& \leq M\left(l L_{2}+L_{3}+\left\|x_{0}\right\|_{\alpha}\right)+\frac{w(l) \sup _{t \in J} K(t) M_{\alpha}}{(q-\alpha) \Gamma(q)} a^{q-\alpha+1}+M \sum_{k=1}^{m} \psi_{k}(l) .
\end{aligned}
$$

Hence $\mathcal{B}$ is bounded on bounded sets of $\Omega$ for each $x \in \mathcal{B}(B(l))$.

Step 4. $B$ sends bounded sets into equicontinuous sets on $\Omega$.

Let $\tau_{1}, \tau_{2} \in J, \tau_{1}<\tau_{2}$. Let $u \in B_{l}$ and $x \in \mathscr{B}(u)$, then there exists $v \in S_{G, u}$ such that, for each $t \in J$, we have

$$
x(t)=T(t)\left(x_{0}-g(x)\right)+\frac{1}{\Gamma(q)} \int_{0}^{t}(t-s)^{q-1} T(t-s) \int_{0}^{s} K(s, x) v(x) d x d s
$$


$+\sum_{k=1}^{m} T\left(t-t_{k}\right) I_{k}\left(x\left(t_{k}^{-}\right)\right)$.

Then

$$
\begin{aligned}
& \left\|x\left(\tau_{2}\right)-x\left(\tau_{1}\right)\right\|_{\alpha} \\
& \leq\left\|\left[T\left(\tau_{2}\right)-T\left(\tau_{1}\right)\right]\left(x_{0}-g(x)\right)\right\|_{\alpha} \\
& +\| \frac{1}{\Gamma(q)} \int_{0}^{\tau_{1}}\left[\left(\tau_{2}-s\right)^{q-1} T\left(\tau_{2}-s\right)-\left(\tau_{1}-s\right)^{q-1} T\left(\tau_{1}-s\right)\right] \\
& +\int_{0}^{s} K(s, x) v(x) d x d s \|_{\alpha} \\
& +\left\|\frac{1}{\Gamma(q)} \int_{\tau_{1}}^{\tau_{2}}\left(\tau_{2}-s\right)^{q-1} T\left(\tau_{2}-s\right) \int_{0}^{s} K(s, x) v(x) d x d s\right\|_{\alpha} \\
& +\left\|\sum_{0<t_{k}<\tau_{1}}\left[T\left(\tau_{2}-t_{k}\right)-T\left(\tau_{1}-t_{k}\right)\right] I_{k}\left(x\left(t_{k}^{-}\right)\right)\right\|_{\alpha} \\
& +\left\|\sum_{\tau_{1}<t<\tau_{2}} T\left(\tau_{2}-t_{k}\right) I_{k}\left(x\left(t_{k}^{-}\right)\right)\right\|_{\alpha} \\
& \leq A_{1}+A_{2}+A_{3}+A_{4}+A_{5}+A_{6},
\end{aligned}
$$

Actually, $A_{1}, A_{2}, A_{3}, A_{4}, A_{5}$ and $A_{6}$ tend to 0 independently of $B_{l}$ when $\tau_{2}-\tau_{1} \rightarrow 0$. Indeed, let $x \in B_{l}$, we have

$$
\begin{aligned}
A_{1} & =\left\|\left[T\left(\tau_{2}\right)-T\left(\tau_{1}\right)\right]\left(x_{0}-g(x)\right)\right\|_{\alpha} \\
& \leq\left\|T\left(\tau_{2}\right)-T\left(\tau_{1}\right)\right\|_{\alpha}\left\|\left(x_{0}-g(x)\right)\right\|_{\alpha} \\
& \leq\left\|T\left(\tau_{2}\right)-T\left(\tau_{1}\right)\right\|_{\alpha}\left(\left\|x_{0}\right\|_{\alpha}+L_{2}\|x\|_{\Omega}+L_{3}\right)
\end{aligned}
$$

from which we deduce that $\lim _{\tau_{2} \rightarrow \tau_{1}} A_{1}=0$ since by Lemma 3 the function $t \rightarrow\|T(t)\|_{\alpha}$ for $t \in(0, a]$.

$$
\begin{aligned}
& A_{2}=\| \frac{1}{\Gamma(q)} \int_{0}^{\tau_{1}}\left[\left(\tau_{2}-s\right)^{q-1}\left[T\left(\tau_{2}-s\right)-T\left(\tau_{1}-s\right)\right] \int_{0}^{s} K(s, x) v(x) d x d s \|_{\alpha}\right. \\
& \leq \frac{a^{q-1}}{\Gamma(q)} \int_{0}^{\tau_{1}}\left\|\left[T\left(\tau_{2}-s\right)-T\left(\tau_{1}-s\right)\right] \int_{0}^{s} K(s, x) v(x)\right\|_{\alpha} d x d s \\
& \leq \frac{a^{q-1}}{\Gamma(q)} \int_{0}^{\tau_{1}} \|\left[T\left(\frac{\tau_{2}-\tau_{1}}{2}+\frac{\tau_{2}-s}{2}\right)-T\left(\frac{\tau_{1}-s}{2}\right)\right] A^{\alpha} T\left(\frac{\tau_{1}-s}{2}\right) \\
& \int_{0}^{s} K(s, x) v(x) \| d x d s
\end{aligned}
$$


$\leq \frac{a^{q-\alpha-1} M_{\alpha} \sup _{t \in J} K(t) w(l)}{\Gamma(q)} \int_{0}^{\tau_{1}}\left[T\left(\frac{\tau_{2}-\tau_{1}}{2}+\frac{\tau_{2}-s}{2}\right)-T\left(\frac{\tau_{1}-s}{2}\right)\right] d s$.

Therefore, the continuity of the function $t \rightarrow\|T(t)\|_{\alpha}$ for $t \in(0, a]$ allows us to conclude that $\lim _{\tau \rightarrow \tau_{1}} A_{2}=0$.

$$
\begin{aligned}
A_{3} & =\left\|\frac{1}{\Gamma(q)} \int_{0}^{\tau_{1}}\left[\left(\tau_{2}-s\right)^{q-1}-\left(\tau_{1}-s\right)^{q-1}\right] T\left(\tau_{1}-s\right) \int_{0}^{s} K(s, x) v(x) d x d s\right\|_{\alpha} \\
& \leq \frac{1}{\Gamma(q)} \int_{0}^{\tau_{1}}\left[\left(\tau_{2}-s\right)^{q-1}-\left(\tau_{1}-s\right)^{q-1}\right]\left\|A^{\alpha} T\left(\tau_{1}-s\right) \int_{0}^{s} K(s, x) v(x) d x\right\| d s \\
& \leq \frac{M_{\alpha} a^{-\alpha}}{\Gamma(q)} \int_{0}^{\tau_{1}}\left[\left(\tau_{2}-s\right)^{q-1}-\left(\tau_{1}-s\right)^{q-1}\right]\left\|\int_{0}^{s} K(s, x) v(x) d x\right\| d s \\
& \leq \frac{M_{\alpha} a^{-\alpha} \sup _{t \in J} w(l)}{\Gamma(q)} \int_{0}^{\tau_{1}}\left[\left(\tau_{2}-s\right)^{q-1}-\left(\tau_{1}-s\right)^{q-1}\right] d s \\
& \leq \frac{M_{\alpha} a^{-\alpha} \sup _{t \in J} w(l)}{q \Gamma(q)}\left|\tau_{2}-\tau_{1}\right|^{q} .
\end{aligned}
$$

Hence $\lim _{\tau_{2} \rightarrow \tau_{1}} A_{3}=0$.

$$
\begin{aligned}
A_{4} & =\left\|\frac{1}{\Gamma(q)} \int_{\tau_{1}}^{\tau_{2}}\left(\tau_{2}-s\right)^{q-1} T\left(\tau_{2}-s\right) \int_{0}^{s} K(s, x) v(x) d x d s\right\|_{\alpha} \\
& \leq \frac{a^{q-1} \sup _{t \in J} K(t) w(l)}{\Gamma(q)} \int_{\tau_{1}}^{\tau_{2}}\left\|A^{\alpha} T\left(\tau_{2}-s\right)\right\| d s \\
& \leq \frac{M_{\alpha} a^{q-1} \sup _{t \in J} K(t) w(l)}{\Gamma(q)} \int_{\tau_{1}}^{\tau_{2}}\left(\tau_{2}-s\right)^{-\alpha} d s \\
& \leq \frac{M_{\alpha} a^{q-1} \sup _{t \in J} K(t) w(l)}{(1-\alpha) \Gamma(q)}\left|\tau_{2}-\tau_{1}\right|^{1-\alpha} .
\end{aligned}
$$

Since $1-\alpha>0$, we deduce that $\lim _{\tau_{2} \rightarrow \tau_{1}} A_{4}=0$.

$$
\begin{aligned}
A_{5} & =\left\|\sum_{0<t_{k}<\tau_{1}}\left[T\left(\tau_{2}-t_{k}\right)-T\left(\tau_{1}-t_{k}\right)\right] I_{k}\left(x\left(t_{k}^{-}\right)\right)\right\|_{\alpha} \\
& \leq \sum_{0<t_{k}<\tau_{1}}\left\|\left[T\left(\tau_{2}-t_{k}\right)-T\left(\tau_{1}-t_{k}\right)\right]\right\|_{\alpha}\left\|I_{k}\left(x\left(t_{k}^{-}\right)\right)\right\|_{\alpha} \\
& \leq \sum_{0<t_{k}<\tau_{1}} \psi_{k}\left(\|x\|_{\alpha}\right)\left\|\left[T\left(\tau_{2}-t_{k}\right)-T\left(\tau_{1}-t_{k}\right)\right]\right\|_{\alpha} .
\end{aligned}
$$

Hence, we deduce $\lim _{\tau_{2} \rightarrow \tau_{1}} A_{5}=0$.

$$
A_{6}=\left\|\sum_{\tau_{1}<t<\tau_{2}} T\left(\tau_{2}-t_{k}\right) I_{k}\left(x\left(t_{k}^{-}\right)\right)\right\|_{\alpha}
$$




$$
\leq \sum_{\tau_{1}<t<\tau_{2}} \psi_{k}\left(\|x\|_{\alpha}\left\|T\left(\tau_{2}-t_{k}\right)\right\|_{\alpha} .\right.
$$

Therefore, $\lim _{\tau_{2} \rightarrow \tau_{1}} A_{6}=0$.

Step 5. $\left(\mathscr{B} B_{l}\right)(t)$ is relatively compact for each $t \in J$, where $\left(\mathscr{B} B_{l}\right)(t)=$

$\left\{x(t) ; x \in \mathscr{B} B_{l}, t \in J\right\}$. Obviously, by condition $(H 3)(i i),\left(\mathscr{B} B_{l}\right)(t)$ is relatively compact in $\Omega$ for $t=0$. Let $0<t \leq a$ be fixed and $0<\epsilon<t$. For $u \in B_{l}$ and $x \in \mathscr{B}(u)$, there exists a function $v \in S_{G, u}$ such that

$$
\begin{gathered}
x(t)=T(t)\left(x_{0}-g(x)\right)+\frac{1}{\Gamma(q)} \int_{0}^{t-\epsilon}(t-s)^{q-1} T(t-s) \int_{0}^{s} K(s, x) v(x) d x d s \\
+\frac{1}{\Gamma(q)} \int_{t-\epsilon}^{t}(t-s)^{q-1} T(t-s) \int_{0}^{s} K(s, x) v(x) d x d s+\sum_{0<t_{k}<t} T\left(t-t_{k}\right) I_{k}\left(x\left(t_{k}^{-}\right)\right) .
\end{gathered}
$$

Define

$$
\begin{gathered}
x_{\epsilon}(t)=T(t)\left(x_{0}-g(x)\right)+\frac{1}{\Gamma(q)} \int_{0}^{t-\epsilon}(t-s)^{q-1} T(t-s) \int_{0}^{s} K(s, x) v(x) d x d s \\
+\sum_{0<t_{k}<t-\epsilon} T\left(t-t_{k}\right) I_{k}\left(x\left(t_{k}^{-}\right)\right) \\
=T(t)\left(x_{0}-g(x)\right)+\frac{T(\epsilon)}{\Gamma(q)} \int_{0}^{t-\epsilon}(t-s)^{q-1} T(t-\epsilon-s) \int_{0}^{s} K(s, x) v(x) d x d s \\
+\sum_{0<t_{k}<t-\epsilon} T\left(t-t_{k}\right) I_{k}\left(x\left(t_{k}^{-}\right)\right) .
\end{gathered}
$$

Since $\{T(t), t>0\}$ is compact, the set $U_{\epsilon}=\left\{x(t): x \in \mathscr{B}\left(B_{l}\right)\right\}$ is relatively compact in $\Omega$ for every $\epsilon, 0<\epsilon<t$. Moreover, for every $x \in \mathscr{B}\left(B_{l}\right)$,

$$
\begin{aligned}
\left\|x(t)-x_{\epsilon}(t)\right\|_{\alpha} & =\frac{1}{\Gamma(q)}\left\|\int_{t-\epsilon}^{t}(t-s)^{q-1} T(t-s) \int_{0}^{s} K(s, x) v(x) d x d s\right\|_{\alpha} \\
& +M \sum_{t-\epsilon<t_{k}<t} \psi(l) .
\end{aligned}
$$

Therefore, letting $\epsilon \rightarrow 0$, we see that, there are relatively compact sets arbitrarily close to the set $\left\{x(t): x \in \mathcal{B}\left(B_{l}\right)\right\}$. Hence the set $x(t) ; x \in \mathcal{B}\left(B_{l}\right)$ is relatively compact in $\Omega$. As a consequence of steps $(3-5)$ together with the Arzela-Ascoli theorem, we can conclude that $\mathscr{B}$ is a compact multivalued map.

Step 6. $B$ has a closed graph.

Let $u_{n} \rightarrow u_{*}, u_{n} \in B_{l},\left\{x_{n} \in \mathscr{B}\left(u_{n}\right)\right\}$, and $x_{n} \rightarrow x_{*}$. We shall prove that $x_{*} \in \mathscr{B}\left(u_{*}\right) . x_{n} \in \mathscr{B}\left(u_{n}\right)$ means that there exists $v_{n} \in S_{G, u_{n}}$ such that, for 
each $t \in J$,

$$
\begin{aligned}
x_{n}(t) & =T(t)\left(u_{0}-g\left(u_{n}\right)\right)+\frac{1}{\Gamma(q)} \int_{0}^{t}(t-s)^{q-1} T(t-s) \int_{0}^{s} K(s, x) v_{n}(x) d x d s \\
& +\sum_{0<t_{k}<t} T\left(t-t_{k}\right) I_{k}\left(u_{n}\left(t_{k}^{-}\right)\right) .
\end{aligned}
$$

We must prove that there exists $v_{*} \in S_{G, u_{*}}$ such that, for each $t \in J$,

$$
\begin{aligned}
x_{*}(t) & =T(t)\left(u_{0}-g\left(u_{*}\right)\right)+\frac{1}{\Gamma(q)} \int_{0}^{t}(t-s)^{q-1} T(t-s) \int_{0}^{s} K(s, x) v_{*}(x) d x d s \\
& +\sum_{0<t_{k}<t} T\left(t-t_{k}\right) I_{k}\left(u_{*}\left(t_{k}^{-}\right)\right) .
\end{aligned}
$$

Clearly since $I_{k}, k=1,2, \cdots, m$ and $g$ are continuous we have that

$$
\begin{aligned}
& \|\left(x_{n}-T(t)\left(u_{0}-g\left(u_{n}\right)\right)-\sum_{0<t_{k}<t} T\left(t-t_{k}\right) I_{k}\left(u_{n}\left(t_{k}^{-}\right)\right)\right) \\
& -\left(x_{*}-T(t)\left(u_{0}-g\left(u_{*}\right)\right)-\sum_{0<t_{k}<t} T\left(t-t_{k}\right) I_{k}\left(u_{*}\left(t_{k}^{-}\right)\right)\right) \|_{\Omega} \rightarrow 0
\end{aligned}
$$

as $n \rightarrow \infty$. Consider the linear continuous operator

$$
\begin{gathered}
\Phi: L^{1}(J, X) \rightarrow \mathcal{C}(J, X) \quad v \rightarrow \Phi(v)(t) \\
=\frac{1}{\Gamma(q)} \int_{0}^{t}(t-s)^{q-1} T(t-s) \int_{0}^{s} K(s, x) v_{n}(x) d x d s
\end{gathered}
$$

From Lemma 1, it follows that $\Phi \circ S_{G, u}$ is a closed graph operator. Moreover we have that

$x_{n}(t)-T(t)\left(u_{0}-g\left(u_{n}\right)\right)-\sum_{0<t_{k}<t} T\left(t-t_{k}\right) I_{k}\left(u_{n}\left(t_{k}^{-}\right)\right) \in \Phi\left(S_{G, u_{n}}\right)$.

Since $u_{n} \rightarrow u_{*}$ it follows from Lemma 1 that

$x_{*}(t)-T(t)\left(u_{0}-g\left(u_{*}\right)\right)-\sum_{0<t_{k}<t} T\left(t-t_{k}\right) I_{k}\left(u_{*}\left(t_{k}^{-}\right)\right) \in \Phi\left(S_{G, u_{*}}\right)$,

that is, there must exist a $v_{*} \in S_{G, u_{*}}$ such that

$$
\begin{aligned}
& x_{*}(t)-T(t)\left(u_{0}-g\left(u_{*}\right)\right)-\sum_{0<t_{k}<t} T\left(t-t_{k}\right) I_{k}\left(u_{*}\left(t_{k}^{-}\right)\right) \\
& =\Phi\left(\left(v_{*}\right)(t)\right)=\frac{1}{\Gamma(q)} \int_{0}^{t}(t-s)^{q-1} T(t-s) \int_{0}^{s} K(s, x) v_{*}(x) d x d s .
\end{aligned}
$$

Therefore, $\mathscr{B}$ has a closed graph and so $\mathscr{B}$ is u.s.c. 
Step 7. The operator inclusion $u \in \mathcal{A}(u)+\mathscr{B}(u)$ has a solution in $B[0, r]$.

Define an open ball $B(0, r)$ in $\Omega$, where the real number $r$ satisfies the inequality given in condition (H6). As a consequence of steps $(1-6)$, we can see that the operators $\mathcal{A}$ and $\mathscr{B}$ satisfy all conditions of Lemma 2. Now we show that the second assertion of Lemma 2 is not true. Let $u \in \Omega$ be a possible solution for $\lambda x \in \mathcal{A} x+\mathscr{B} x$ for some $\lambda>1$ with $\|x\|_{\alpha}=r$. Then we have,

$$
\begin{aligned}
x(t) & =\lambda^{-1} T(t)\left[u_{0}-g(u)-F\left(0, u\left(h_{1}(0)\right)\right)\right]+\lambda^{-1} F\left(t, u\left(h_{1}(t)\right)\right) \\
& +\frac{\lambda^{-1}}{\Gamma(q)} \int_{0}^{t}(t-s)^{q-1} T(t-s) F\left(s, x\left(h_{1}(s)\right)\right) d s \\
& +\frac{\lambda^{-1}}{\Gamma(q)} \int_{0}^{t}(t-s)^{q-1} T(t-s) \int_{0}^{s} K(s, x) v(x) d w d s \\
& +\lambda^{-1} \sum_{k=1}^{m} T\left(t-t_{k}\right) I_{k}\left(u\left(t_{k}^{-}\right)\right) .
\end{aligned}
$$

Thus by $(H 1)-(H 5)$

$$
\begin{aligned}
\|x(t)\|_{\alpha} & \leq \lambda^{-1}\left\|T(t)\left[u_{0}-g(u)-F\left(0, u\left(h_{1}(0)\right)\right)\right]\right\|_{\alpha}+\lambda^{-1}\left\|F\left(t, u\left(h_{1}(t)\right)\right)\right\|_{\alpha} \\
& +\frac{\lambda^{-1}}{\Gamma(q)} \int_{0}^{t}\left\|(t-s)^{q-1} T(t-s) F\left(s, x\left(h_{1}(s)\right)\right) d s\right\|_{\alpha} \\
& +\frac{\lambda^{-1}}{\Gamma(q)} \int_{0}^{t}\left\|(t-s)^{q-1} T(t-s) \int_{0}^{s} K(s, x) v(x) d w d s\right\|_{\alpha} \\
& +\lambda^{-1} \sum_{k=1}^{m}\left\|T\left(t-t_{k}\right) I_{k}\left(u\left(t_{k}^{-}\right)\right)\right\|_{\alpha} \\
& \leq I_{1}+I_{2}+I_{3}+I_{4}+I_{5}
\end{aligned}
$$

where,

$$
\begin{gathered}
I_{1}=\lambda^{-1}\left\|T(t)\left[u_{0}-g(u)-F\left(0, u\left(h_{1}(0)\right)\right)\right]\right\|_{\alpha} \\
\leq M\left\|u_{0}-g(u)\right\|_{\alpha}+\left\|A^{\alpha} F\left(0, u\left(h_{1}(0)\right)\right)\right\| \\
\leq M\left(\left\|u_{0}\right\|_{\alpha}+\|g(u)\|_{\alpha}\right)+\left(L_{1}\|u\|_{\Omega}+1\right) \\
\leq M\left(\left\|u_{0}\right\|_{\alpha}+L_{2}\|u\|_{\Omega}+L_{3}\right)+\left(L_{1}\|u\|_{\Omega}+1\right) . \\
I_{2}=\lambda^{-1}\left\|F\left(t, u\left(h_{1}(t)\right)\right)\right\|_{\alpha} \\
\leq\left\|A^{\alpha} F\left(t, u\left(h_{1}(t)\right)\right)\right\| \\
\leq\left(L_{1}\|u\|_{\Omega}+1\right) .
\end{gathered}
$$




$$
\begin{aligned}
& I_{3}=\frac{\lambda^{-1}}{\Gamma(q)} \int_{0}^{t}\left\|(t-s)^{q-1} T(t-s) F\left(s, x\left(h_{1}(s)\right)\right) d s\right\|_{\alpha} \\
& \leq \frac{1}{\Gamma(q)} \int_{0}^{t}\left\|(t-s)^{q-1} A^{\alpha} T(t-s) F\left(s, x\left(h_{1}(s)\right)\right) d s\right\| \\
& \leq \frac{M_{\alpha}\left(L_{1}\|u\|_{\Omega}+1\right)}{\Gamma(q)} \int_{0}^{t}(t-s)^{q-1-\alpha} d s \\
& \leq \frac{M_{\alpha}\left(L_{1}\|u\|_{\Omega}+1\right) a^{q-\alpha}}{\Gamma(q)(q-\alpha)} \\
& I_{4}=\frac{\lambda^{-1}}{\Gamma(q)} \int_{0}^{t}\left\|(t-s)^{q-1} T(t-s) \int_{0}^{s} K(s, x) v(x) d w d s\right\|_{\alpha} \\
& \leq \frac{a w\left(\|u\|_{\alpha}\right) \sup _{t \in J} K(t)}{\Gamma(q)} \int_{0}^{t}(t-s)^{q-1}\left\|A^{\alpha} T(t-s)\right\| d s \\
& \leq \frac{w\left(\|u\|_{\alpha}\right) M_{\alpha} a^{q-\alpha+1} \sup _{t \in J} K(t)}{(q-\alpha) \Gamma(q)} \\
& I_{5}=\lambda^{-1} \sum_{k=1}^{m}\left\|T\left(t-t_{k}\right) I_{k}\left(u\left(t_{k}^{-}\right)\right)\right\|_{\alpha} \\
& \leq \sum_{k=1}^{m}\left\|T\left(t-t_{k}\right) I_{k}\left(u\left(t_{k}^{-}\right)\right)\right\|_{\alpha} \\
& \leq M \sum_{k=1}^{m} \psi_{k}\left(\|u\|_{\alpha}\right) .
\end{aligned}
$$

Taking the supremum over $t$ we obtain,

$$
\begin{gathered}
\|x(t)\|_{\Omega} \leq M\left(\left\|u_{0}\right\|_{\alpha}+L_{2}\|u\|_{\Omega}+L_{3}\right)+\left(L_{1}\|u\|_{\Omega}+1\right)+\left(L_{1}\|u\|_{\Omega}+1\right) \\
+\frac{M_{\alpha}\left(L_{1}\|u\|_{\Omega}+1\right) a^{q-\alpha}}{\Gamma(q)(q-\alpha)}+\frac{w\left(\|u\|_{\Omega}\right) M_{\alpha} a^{q-\alpha+1} \sup _{t \in J} K(t)}{(q-\alpha) \Gamma(q)} \\
+M \sum_{k=1}^{m} \psi_{k}\left(\|u\|_{\Omega}\right) .
\end{gathered}
$$

Substituting $\|u\|_{\Omega}=r$ in the above inequality and noting that (3.2) holds, we have

$$
\begin{aligned}
r & \leq M\left(\left\|u_{0}\right\|_{\alpha}+L_{2} r+L_{3}\right)+\left(L_{1} r+1\right)+\left(L_{1} r+1\right)+\frac{M_{\alpha}\left(L_{1} r+1\right) a^{q-\alpha}}{\Gamma(q)(q-\alpha)} \\
& +\frac{w(r) M_{\alpha} a^{q-\alpha+1} \sup _{t \in J} K(t)}{(q-\alpha) \Gamma(q)}+M \sum_{k=1}^{m} \psi_{k}(r)
\end{aligned}
$$


and hence

$$
r \leq \frac{2+M\left(\left\|u_{0}\right\|_{\alpha}+L_{3}\right)+\frac{M_{\alpha} a^{q-\alpha}(1+a w(r) K)}{(q-\alpha) \Gamma(q)}}{1-\left[M\left(L_{2}+m N\right)+2 L_{1}+\frac{M_{\alpha} L_{1} a^{q-\alpha}}{(q-\alpha) \Gamma(q)}\right]}
$$

which is contradiction to (3.2). Hence the operator inclusions $u \in \mathcal{A}(u)+$ $\mathscr{B}(u)$ has a solution in $B[0, r]$. It further implies that the system (1.1) has at least one mild solution $\mathrm{x}$ in $\Omega$. The proof is complete.

\section{AN EXAMPLE}

As an application of previous sections, we study the fractional neutral differential equation of order $q$, where $0<q \leq 1$.

$$
\begin{aligned}
& \frac{\partial^{q}}{\partial t^{q}}\left[u(t, x)-\int_{0}^{\pi} \Theta(t, y, x)\left[u(\sin t, y)+\frac{\partial}{\partial y} u(\sin t, y)\right] d y\right] \\
& =\frac{\partial^{2}}{\partial x^{2}} u(t, x)+\int_{0}^{s} K(t, s) g\left(t, u(\sin t, x), \frac{\partial}{\partial x} u(\sin t, x)\right) d s, \\
& 0 \leq t \leq 1,0 \leq x \leq \pi 0 \leq s \leq t, t \neq t_{k}, \quad k=1, \cdots, m \\
& u(t, 0)=u(t, \pi)=0 . \\
& u\left(t_{k}^{+}\right)-u\left(t_{k}^{-}\right)=I_{k}\left(u\left(t_{k}^{-}\right)\right) \quad k=1, \cdots, m \\
& u(0, x)+\sum_{i=0}^{p} \int_{0}^{\pi} k_{i}(y, x) u\left(s_{i}, y\right) d y=u_{0}(x), \quad 0 \leq x \leq \pi,
\end{aligned}
$$

where $0<q \leq 1,0<t_{1}<\cdots, t_{p}, u_{0} \in X=L^{2}([0,1], \mathbb{R})$ equipped with the $L^{2}$-norm $\|\cdot\|_{2}$ and $k_{i}(.,.) \in L^{2}([0,1] \times[0,1], \mathbb{R})$ for $i=1,2$.

Consider the operator $A: D(A) \subset X \rightarrow X$ defined by

$$
\left\{\begin{array}{l}
\left.D(A)=H_{0}^{2}([0, \pi)]\right)=\left\{f \in X: f, f^{\prime \prime} \in X, f(0)=f(\pi)=0\right\}, \\
A f=-f^{\prime \prime} .
\end{array}\right.
$$

Then $-A$ generates a compact, analytic semigroup $T($.$) of uniformly bounded linear$ operators. It is well known that $0 \in \rho(A)$ and so the fractional powers of $A$ are well defined. Moreover, the eigenvalues of $A$ are $n^{2}$ and the corresponding normalized eigenvectors are $e_{n}(x)=\sqrt{\frac{2}{\pi}} \sin (n x), n=1,2, \cdots$. The following results are well known:

(A1) If $z \in D(A)$, then

$$
A z=\sum_{n=1}^{\infty} n^{2}\left\langle z, e_{n}\right\rangle e_{n}
$$


(A2) For each $z \in X$,

$$
A^{-\frac{1}{2}} z=\sum_{n=1}^{\infty} \frac{1}{n}\left\langle z, e_{n}\right\rangle e_{n} .
$$

(A3) The operator $A^{\frac{1}{2}}$ is given by

$$
A^{\frac{1}{2}} z=\sum_{n=1}^{\infty} n\left\langle z, e_{n}\right\rangle e_{n}
$$

for each $z \in D\left(A^{\frac{1}{2}}\right):=\left\{f \in X: \sum_{n=1}^{\infty} n\left\langle z, e_{n}\right\rangle e_{n} \in X\right\}$.

We assume that the following conditions hold:

(i) The function $\Theta$ is measurable and

$$
\sup _{0 \leq t \leq 1} \int_{0}^{\pi} \int_{0}^{\pi} \Theta^{2}(t, y, x) d y d x<\infty .
$$

(ii) For each $t \in J, K(t, s)$ is measurable on $J$ and

$$
K(t)=e s s \sup \{|K(t, s)|, 0 \leq s \leq t\} \text {, is bounded on } J .
$$

(iii) The function $\frac{\partial^{2}}{\partial x^{2}}$ is measurable, $\Theta(t, y, 0)=\Theta(t, y, \pi)=0$, and

$$
\sup _{0 \leq t \leq 1}\left[\int_{0}^{\pi} \int_{0}^{\pi}\left(\frac{\partial^{2}}{\partial x^{2}} \Theta^{2}(t, y, x)\right)^{2} d y d x\right]^{\frac{1}{2}}<\infty .
$$

(iv) For the function $g: J \times \mathbb{R} \times \mathbb{R} \rightarrow \mathbb{R}$ the following three conditions are satisfied:

(a) For each $t \in J, g(t, .,$.$) is continuous.$

(b) For each $u \in X_{\frac{1}{2}}, g\left(., u, u^{\prime}\right)$ is measurable.

(c) There is a positive number $v$ such that

$$
\left\|g\left(t, u, u^{\prime}\right)\right\| \leq v\|u\|, \quad \text { for all }(t, u) \in J \times X_{\frac{1}{2}} .
$$

(v) $I_{k} \in \mathcal{C}\left(X_{\alpha}, X_{\alpha}\right), k=1,2, \cdots, m$ and there exist nondecreasing function $\psi_{k} \in$ $\mathcal{C}\left(J, \mathbb{R}_{+}\right)$such that for each $u \in X_{\alpha}$

$$
\left\|I_{k}(u)\right\| \leq \psi_{k}\left(\|u\|_{\alpha}\right) .
$$

Here we choose $\alpha=\beta=\frac{1}{2}$. It follows from [15] that if $u \in X_{\frac{1}{2}}$, then $u$ is absolutely continuous, $u^{\prime} \in X$, and $u(0)=u(\pi)=0$. In view of this result, for $(t, u) \in J \times X_{\frac{1}{2}} w \in \Omega$, we can define respectively that

$F(t, u)(x)=\int_{0}^{\pi} \Theta(t, y, x)\left[u(y)+u^{\prime}(y)\right] d y^{\prime}$,

$G(t, u)(x)=g\left(t, u(x), u^{\prime}(x)\right) \quad$ and 
$g(w(t))=\sum_{i=0}^{p} K_{i}(u)(x) w\left(t_{i}\right)=\sum_{i=0}^{p} \int_{0}^{\pi} k_{i}(y, x) u(y) d y, \quad w \in \Omega$.

So $G: J \times X_{\frac{1}{2}} \rightarrow X$. And it is easy to see that $F: J \times X_{\frac{1}{2}} \rightarrow X_{\frac{1}{2}}, A^{\frac{1}{2}} F:$ $J \times X_{\frac{1}{2}} \rightarrow X_{\frac{1}{2}}^{2}$. In fact, for each $t \in J$, we have

$\left\langle F(t, u), u_{n}\right\rangle=\frac{1}{n} \sqrt{\frac{2}{\pi}}\left(\int_{0}^{\pi} \frac{\partial}{\partial x} \Theta(t, y, x)\left[u(y)+u^{\prime}(y)\right] d y, \cos (n \pi)\right)$

and also

$\left\langle A^{\frac{1}{2}} F(t, u), u_{n}\right\rangle=\frac{1}{n^{2}} \sqrt{\frac{2}{\pi}}\left(\int_{0}^{\pi} \frac{\partial^{2}}{\partial x^{2}} \Theta(t, y, x)\left[u(y)+u^{\prime}(y)\right] d y, \sin (n \pi)\right)$.

It shows that $F$ and $A^{\frac{1}{2}} F$ both take values in $X_{\frac{1}{2}}$ in terms of properties $A 1$ and $A 3$, and so does the function $g$. Since, for any $x_{1}, x_{2} \in X_{\frac{1}{2}}$,

$$
\begin{aligned}
\left\|x_{2}-x_{1}\right\|^{2} & =\sum_{n=1}^{\infty}\left\langle x_{2}-x_{1}, e_{n}\right\rangle^{2} \\
& \leq \sum_{n=1}^{\infty} n^{2}\left\langle x_{2}-x_{1}, e_{n}\right\rangle^{2} \\
& \leq\left\|x_{2}-x_{1}\right\|_{\frac{1}{2}} .
\end{aligned}
$$

from which and conditions (iii) we see that $(H 1)$ and $(H 2)$ are satisfied. In addition, $G$ satisfies condition $(H 4)$ while $g$ verifies $(H 5)$. From (i) it is clear that $F(t, u)$ is bounded linear operator on $X$. Let $h_{1}(t)=h_{2}(t)=\sin t$. If conditions (3.1)-(3.3) are satisfied, then from Theorem 2, system (4.1) admits a mild solution on $[0,1]$ under the above assumptions.

\section{ACKNOWLEDGEMENT}

The authors wish to thank the referee for useful comments and suggestions.

\section{REFERENCES}

[1] D. D. Bainov and P. S. Simeonov, Systems with impulse effect. Chichester: Ellis Horwood, 1989.

[2] L. Byszewski, "Theorems about the existence and uniqueness of solutions of a semilinear evolution nonlocal Cauchy problem," J. Math. Anal. Appl., vol. 162, no. 2, pp. 494-505, 1991.

[3] L. Byszewski and H. Akca, "Existence of solutions to a semilinear functional-differential evolution nonlocal problem," Nonlinear Anal., Theory Methods Appl., vol. 34, no. 1, pp. 65-72, 1998.

[4] L. Byszewski and V. Lakshmikantham, "Theorem about the existence and uniqueness of a solution of a nonlocal abstract Cauchy problem in a Banach space," Appl. Anal., vol. 40, no. 1, pp. 11-19, 1991. 
[5] Y.-K. Chang, A. Anguraj, and K. Karthikeyan, "Existence for impulsive neutral integrodifferential inclusions with nonlocal initial conditions via fractional operators," Nonlinear Anal., Theory Methods Appl., Ser. A, Theory Methods, vol. 71, no. 10, pp. 4377-4386, 2009.

[6] K. Deimling, Multivalued differential equations, ser. e Gruyter Studies in Nonlinear Analysis and Applications. Berlin: Walter de Gruyter, 1992, vol. 1.

[7] K. Deng, "Exponential decay of solutions of semilinear parabolic equations with nonlocal initial conditions," J. Math. Anal. Appl., vol. 179, no. 2, pp. 630-637, 1993.

[8] B. Dhage, "Multi-valued mappings and fixed points. II," Tamkang J. Math., vol. 37, no. 1, pp. 27-46, 2006.

[9] K. Ezzinbi, X. Fu, and K. Hilal, "Existence and regularity in the $\alpha$-norm for some neutral partial differential equations with nonlocal conditions," Nonlinear Anal., Theory Methods Appl., Ser. A, Theory Methods, vol. 67, no. 5, pp. 1613-1622, 2007.

[10] X. Fu, "On solutions of neutral nonlocal evolution equations with nondense domain," J. Math. Anal. Appl., vol. 299, no. 2, pp. 392-410, 2004.

[11] X. Fu and K. Ezzinbi, "Existence of solutions for neutral functional differential evolution equations with nonlocal conditions," Nonlinear Anal., Theory Methods Appl., Ser. A, Theory Methods, vol. 54, no. 2, pp. 215-227, 2003.

[12] V. Lakshmikantham, D. Bajnov, and P. Simeonov, Theory of impulsive differential equations, ser. Series in Modern Applied Mathematics. Singapore: World Scientific, 1989, vol. 6.

[13] A. Lasota and Z. Opial, "An application of the Kakutani-Ky Fan theorem in the theory of ordinary differential equations,” Bull. Acad. Pol. Sci., Ser. Sci. Math. Astron. Phys., vol. 13, pp. 781-786, 1965.

[14] H. Liu and J.-C. Chang, "Existence for a class of partial differential equations with nonlocal conditions," Nonlinear Anal., Theory Methods Appl., Ser. A, Theory Methods, vol. 70, no. 9, pp. 3076-3083, 2009.

[15] K. S. Miller and B. Ross, An introduction to the fractional calculus and fractional differential equations. New York: John Wiley \& Sons, Inc., 1993.

[16] A. Pazy, Semigroups of linear operators and applications to partial differential equations, ser. Applied Mathematical Sciences. New York: Springer-Verlag, 1983, vol. 44.

[17] A. Samoilenko and N. Perestyuk, Impulsive differential equations. Transl. from the Russian by Yury Chapovsky, ser. World Scientific Series on Nonlinear Science. Series A. Singapore: World Scientific, 1995, vol. 14.

[18] K. Yosida, Functional analysis. 6th ed, ser. Grundlehren der mathematischen Wissenschaften. Berlin-Heidelberg-New York: Springer-Verlag, 1980, vol. 123.

Authors' addresses

M. H. M. Rashid

Department of Mathematics, Statistics, Faculty of Science, Mutah University, P.O. Box(7) AlkarakJordan

E-mail address: malik_okasha@yahoo.com

A. Al-Omari

Department of Mathematics, Faculty of Sciences, Al al-Bayt University, P.O. Box 130095, Mafraq 25113, Jordan

E-mail address: omarimutah1@yahoo.com 